\title{
1 Prediction and Structural Uncertainty Analyses of Artificial Neural Networks Using \\ 2 Hierarchical Bayesian Model Averaging
}

$6 \quad{ }^{1}$ Formerly, Graduate Student, Department of Civil and Environmental Engineering, Louisiana

7 State University, 3418G Patrick F. Taylor Hall, Baton Rouge, LA 70803, USA. Tel: 1-225-578-

8 4246; Fax: 1-225-578-4945; Email: nchits2@1su.edu

$10{ }^{2}$ Assistant Professor, Department of Geology, Faculty of Science, University of Tabriz, 29

11 Bahman Boulevard, Tabriz, East Azarbaijan, Iran, Post code: 5166616471. Tel: 04113392731;

12 Fax: 0411335029; Email: Nadiri@ tabrizu.ac.ir

13

$14{ }^{3}$ Associate Professor, Department of Civil and Environmental Engineering, Louisiana State 15 University, 3418G Patrick F. Taylor Hall, Baton Rouge, LA 70803, USA. Tel: 1-225-578-4246;

16 Fax: 1-225-578-4945; Email: ftsai@1su.edu

17

$18 *$ Corresponding Author 
This study adopts a hierarchical Bayesian model averaging (HBMA) method to analyze

22 prediction uncertainty resulted from uncertain components in artificial neural networks (ANNs).

23 The HBMA is an ensemble method for prediction and is used to segregate the sources of model

24 structure uncertainty in ANNs and investigate their variance contributions to total prediction

25 variance. Specific sources of uncertainty considered in ANNs include the uncertainty in neural

26 network weights and biases (model parameters), uncertainty of selecting an activation function

27 for the hidden layer, and uncertainty of selecting a number of hidden layer nodes (model

28 structure). Prediction uncertainties due to uncertain inputs and ANN model parameters are

29 represented by within-model variance. Prediction uncertainties due to uncertain activation

30 function and uncertain number of nodes for the hidden layer are represented by between-model

31 variance. The method is demonstrated through a study that employs ANNs to predict fluoride

32 concentration in the aquifers of the Maku area, Azarbaijan, Iran. The results show that uncertain

33 inputs and ANN model parameters produces the most prediction variance, followed by

34 prediction variances from uncertain number of hidden layer nodes and uncertain activation

35 function.

37 Keywords: Bayesian model averaging, artificial neural network, uncertainty, ensemble method 


\section{Introduction}

Artificial neural networks (ANNs) are mathematical approximators with strong ability to find the non-linear correlation between input and output data without requiring a comprehensive

41 knowledge about the physical system (Zhang et al., 2009). ANNs have been applied to various

42 hydrologic problems (Govindaraju, 2000a; b; Dawson and Wilby, 2001), for example river flood

43 forecasting (Sudheer et al., 2003; Wei et al., 2012), rainfall forecasting (Hsu et al., 1995; Kumar

44 et al., 2005; Valverde Ramírez et al., 2005), evaporation modeling (Sudheer et al., 2002),

45 groundwater level modeling (Coppola et al., 2005; Daliakopoulos et al., 2005; Nourani et al.,

46 2008a,b; Jha and Sahoo, 2015), and groundwater remediation optimization (Rogers and Dowla,

47 1994). Despite wide applications of ANNs in the field of hydrology, over-parameterization in

48 weights and biases and consequently overfitting to "in-sample" data is a major limitation of

49 ANNs, which may occur in the process of maximizing likelihood functions or alternatively

50 minimizing fitting errors (Kingston et al., 2008). This may reduce prediction accuracy for "out-

51 sample" data that are not used for training ANNs (Tetko et al., 1995; Maier and Dandy, 2000). In

52 other words, overfitting can reduce the "generalization" ability of ANN models. Generalization

53 is the ability of an ANN model to represent an underlying system for an "out-sample" data that

54 are not used in the training procedure. Therefore, it is crucial to select an ANN structure based

55 on the parsimony principle (Box, 1976; Stone, 1981) to balance fitting errors and the number of

56 unknown parameters. To address this issue, Franses and Draisma, (1997) proposed the use of

57 information criteria to choose the best ANN structure. Information criteria tend to select a

58 simpler ANN structure unless a more complicated structure provides a better understanding of

59 the underlying system. However, Qi and Zhang (2001) empirically showed that the selected

60 ANN based on information criteria does not necessarily have an acceptable performance for 
61 fitting "out-sample" data. They concluded that "to improve generalization performance of neural

62 network models, one may need to go beyond the model selection methods".

An alternative to the model selection approach is the artificial neural network ensemble

64 (Shu and Burn, 2004). In this approach, outputs from several ANN models, which are trained by

65 the same data, are combined to generate an ensemble output. Several methods for combining the

66 outputs of multiple artificial intelligence models are proposed in the literature (Ahmad and

67 Zhang, 2002; Shu and Burn, 2004), such as the committee machine (CM) (Perrone and Cooper,

68 1993; Wong et al., 2000; Chen and Lin, 2006; Kadkhodaie-Ilkhchi et al. 2009; Labani et al.

69 2010), the supervised committee machine (SCM) (Drucker, 1997; Hu and Tsoukalas, 2003;

70 Nadiri et al., 2013), and the Bayesian model averaging (BMA) (Nadiri et al., 2014). Although the

71 committee machine methods are shown to improve the generalization ability of ANNs (Shu and

72 Burn, 2004), they are subjected to two major shortcomings. First, they are not able to quantify

73 the prediction uncertainty. Second, they do not consider the parsimony principle in evaluating

74 the combination of individual ANN models.

ANN prediction uncertainty stems from two main sources: (1) the uncertainty in ANN

76 inputs, weights and biases and (2) the uncertainty in ANN structures. Bayesian neural networks

77 (BNNs) (Bishop, 1995; Neal, 1995; Müller and Insua, 1998) were used to train ANN models and

78 to quantify uncertainties from ANN weights and biases (Kingston et al., 2005; Khan and

79 Coulibaly, 2006) as well as uncertainties from model structures (Zhang et al., 2009). The BNN

80 approach prescribes ANN parameters by relatively broad prior probability distributions. Then,

81 during the calibration (training) period, prior distributions are sampled by Markov chain Monte

82 Carlo (MCMC) (Geyer, 1992) or Metropolis algorithm (Haario et al., 2001) to update to

83 posterior distributions given observation data. Finally, the posterior distributions of ANN 
84 parameters are used to evaluate prediction means and variances. However, the BNN method can

85 be overwhelmed by a high number of ANN parameters. In addition, it is crucial to identify the

86 contribution of individual sources of uncertainty to total prediction uncertainty (Wagener and

87 Gupta, 2005).

88 In this study, we adopt the hierarchical Bayesian model averaging (HBMA) (Chitsazan

89 and Tsai 2015a; Tsai and Elshall, 2013) to analyze the contribution of individual sources of

90 uncertainty to ANN predictions. Bayesian model averaging (Draper, 1995; Hoeting et al., 1999)

91 is a well-known ensemble approach based on the law of total probabilty and Bayes' theorem.

92 BMA derives overall prediction mean and variance by averaging all models at once (Nadiri et al.

93 2014). However, it does not quantify contributions of individual uncertain model elements to

94 total estimated variance. The HBMA extends the Bayesian model averaging method by

95 segregating multiple sources of conceptual model structure uncertainty in a hierarchical order

96 (Chitsazan and Tsai, 2015b). The HBMA derives BMA prediction mean and variance by

97 averaging subset models that are developed by an uncertainty source. By doing so, the HBMA is

98 able to identify the contribution of each source of uncertainty to total prediction variance. This

99 study adopts the HBMA to analyze the structural uncertainty as well as parameter uncertainty in

100 the ANNs. The advantages of the HBMA in comparison to other neural network ensemble

101 methods are investigated via the study of predicting fluoride concentration in aquifers of the

102 Maku area, Azarbaijan, Iran.

103 2. Methodology

$104 \quad 2.1$ Artificial Neural Network (ANN)

105 Artificial Neural Networks (ANNs) have been applied to various large-scale problems,

106 including pattern recognition, classification, parameter estimation and prediction (Anderson and 
107 McNeill, 1992; Ablameyko, 2003). The most widely used ANNs to simulate hydrological 108 systems are the multilayer perceptron (MLP), a three-layer feedforward neural network 109 (Govindaraju, 2000 a,b; Sharma et al., 2003). The MLP consists of an input layer, a hidden layer, 110 and an output layer. Figure 1 shows a schematic of a typical multilayer perceptron ANN

111 structure. This study uses four types of input data $\left(\mathrm{Na}^{+}, \mathrm{K}^{+}, \mathrm{Ca}^{2+}, \mathrm{HCO}_{3}^{-}\right)$. The processing

112 elements in each layer are called neurons or nodes. The input layer consists of input variables for 113 the network. The output layer sends out predictions (outputs) of the network. The output function 114 of the output layer is a linear function that aggregates the input signals of the output layer. The 115 hidden layers are placed between the input layer and the output layer to transform and deliver 116 signals. A vital part of the hidden layer which has a main role in transferring the network inputs 117 to output is an activation function, which is typically a continuous and bounded nonlinear 118 function such as hyperbolic-tangent-sigmoid (Tansig) or logarithm-sigmoid (Logsig) functions 119 (Cybenko, 1989).

120 Training algorithms are needed in order to train ANNs to behave similar to the system of 121 interest. Data used for training ANNs are referred to as "in-sample" data. Data that are not used 122 in the training procedure is referred to as "out-sample" data. A brief overview of the MLP, 123 including its mathematical aspects and implementation details, can be found in Govindaraju 124 (2000a). The mathematical expression of a three-layer feedforward ANN for prediction is

$125 O_{j k}=f_{1}\left(b_{j}+\sum_{i} W_{i j} I_{i k}\right)$

$126 O_{k}=b+\sum_{j} W_{j} O_{j k}$

127 where $f_{1}$ is the activation function for the hidden layer, $I_{i k}$ is the $i^{\text {th }}$ input for the $\mathrm{k}^{\text {th }}$ sample 128 point, $O_{j k}$ is the output of $\mathrm{j}^{\text {th }}$ node of the hidden layer, $W_{i j}$ and $W_{j}$ are the weights that control the 
129 strength of connections between layers and $b_{j}$ and $b$ are the biases that are used to adjust the

130 mean value for the hidden layer and the output layer, respectively. The ANN output $O_{k}$ is the $\mathrm{k}^{\text {th }}$

131 predicted fluoride concentration. In the ANN training step, we use the Levenberg-Marquardt

132 (LM) algorithm (Sahoo and Ray, 2006; Sahoo et al., 2006) as a supervised learning algorithm to

133 estimate the weights $\left(W_{i j}\right.$ and $\left.W_{j}\right)$ and the biases ( $b_{j}$ and $b$ ) (Daliakopoulos et al., 2005).

134 There are two common sources of uncertainty in the three-layer feedforward ANN

135 structure: (1) which activation function should be used for the hidden layer? (2) how many nodes

136 are needed in the hidden layer? This study considers Tansig and Logsig activation functions and

137 two to nine nodes in the hidden layer, combinations of which result in $2 \times 8=16$ ANN structures

138 for fluoride concentration prediction.

$139 \quad$ 2.2 Hierarchical Bayesian Model Averaging for Neural Networks

140 To segregate and prioritize the sources of structural uncertainty in ANNs, this study

141 introduces the Hierarchical Bayesian Model Averaging (HBMA) for prediction and uncertainty

142 analysis. Consider $p$ sources of uncertainty in a hierarchical order. Each uncertainty source may

143 suggest a number of propositions (or alternatives). For example, in this study the uncertainty in

144 choosing an activation function for the hidden layer suggests two propositions: Tansig function

145 and Logsig function. The uncertainty in choosing the number of nodes in the hidden layer

146 suggests eight propositions: from two nodes to nine nodes. The combinations of all considered

147 sources of structural uncertainty form the base models for fluoride concentration prediction.

148 We segregate and prioritize the sources of uncertainty in a hierarchical order and denote

149 the base models at level $\mathrm{p}$ and name this level as base level. Aggregation of base models through

150 the BMA over different propositions under the same source of uncertainty forms BMA models at 151 one level up, named level p-1. Aggregation of BMA models at level p-1 through the BMA over 
152 different propositions under the same source of uncertainty forms another BMA models at one

153 level up, named level p-2. Aggregating models continues until the final BMA model, named the

154 hierarch model, is obtained at level 0, which is named the hierarch level. As a result, a BMA tree

155 structured by the base models and the BMA models is developed. In the BMA tree, a parent

156 model is a model in a level with respect to its child models immediately one level below.

157 Using the law of total probability (Yates and Goodman, 1999), given a set of data $\mathbf{D}$ the 158 posterior probability of estimated fluoride concentration $F$ at level $n$ can be obtained as follows

159 (Chitsazan and Tsai, 2015a,b)

160

$$
\begin{aligned}
\operatorname{Pr}\left(F \mid \mathbf{D}, \mathbf{M}_{n}\right) & =\mathrm{E}_{\mathbf{M}_{n+1}}\left[\operatorname{Pr}\left(F \mid \mathbf{D}, \mathbf{M}_{n+1}\right)\right] \\
& =\sum_{q} \mathrm{E}\left(\operatorname{Pr} \mid \mathbf{D}, \mathbf{M}_{n+1}^{(q)}\right) \operatorname{Pr}\left(\mathbf{M}_{n+1}^{(q)} \mid \mathbf{D}, \mathbf{M}_{n}\right)
\end{aligned}
$$

161 where $\mathrm{E}_{\mathbf{M}_{n+1}}$ is the expectation operator over child models $\mathbf{M}_{n+1}^{(q)}$ at level $\mathrm{n}+1$ under their parent

162 model $\mathbf{M}_{n}, \operatorname{Pr}\left(F \mid \mathbf{D}, \mathbf{M}_{n+1}\right)$ is the posterior probability of the fluoride concentration prediction

$163 F$ given data $\mathbf{D}$ and base models $\mathbf{M}_{n+1}$ at level $\mathrm{n}+1$, and $\operatorname{Pr}\left(\mathbf{M}_{n+1}^{(q)} \mid \mathbf{D}, \mathbf{M}_{n}\right)$ is the conditional

164 posterior probability for model $\mathbf{M}_{n+1}^{(q)}$ under their parent models $\mathbf{M}_{n}$ given data $\mathbf{D}$. It is important

165 to recognize that $\mathbf{M}_{n+1}^{(q)}$ is a child model of $\mathbf{M}_{n}$ in $\operatorname{Pr}\left(\mathbf{M}_{n+1}^{(q)} \mid \mathbf{D}, \mathbf{M}_{n}\right)$.

166 When $\mathrm{n}=\mathrm{p}, \mathbf{M}_{p}$ is the set of the ANN models, the base models. Using equation (3) as a

167 recursive equation, the posterior probability of estimated fluoride concentration prediction at 168 level $\mathrm{n}$ is

$169 \operatorname{Pr}\left(F \mid \mathbf{D}, \mathbf{M}_{n}\right)=\mathrm{E}_{\mathbf{M}_{n+1}} \mathrm{E}_{\mathbf{M}_{n+2}} \cdots \mathrm{E}_{\mathbf{M}_{p}}\left[\operatorname{Pr}\left(F \mid \mathbf{D}, \mathbf{M}_{p}\right)\right]$

170 where $\mathrm{E}_{\mathbf{M}_{n+1}} \mathrm{E}_{\mathbf{M}_{n+2}} \cdots \mathrm{E}_{\mathbf{M}_{p}}$ represents a series of averaging operations over the models from level $171 \quad p$ up to level $\mathrm{n}+1$. 
173 prediction at level $n$ is

$174 \mathrm{E}\left(F \mid \mathbf{D}, \mathbf{M}_{n}\right)=\mathrm{E}_{\mathbf{M}_{n+1}}\left[\mathrm{E}\left(F \mid \mathbf{D}, \mathbf{M}_{n+1}\right)\right]$,

175 where $\mathrm{E}\left(F \mid \mathbf{D}, \mathbf{M}_{n+1}\right)$ is the expected value of prediction for given data $\mathbf{D}$ and models at level

$176 \mathrm{n}+1$. According to the law of total variance, the variance of fluoride concentration prediction at

177 level $n<p$ is

$178 \operatorname{Var}\left(F \mid \mathbf{D}, \mathbf{M}_{n}\right)=\mathrm{E}_{\mathbf{M}_{n+1}}\left[\operatorname{Var}\left(F \mid \mathbf{D}, \mathbf{M}_{n+1}\right)\right]+\operatorname{Var}_{\mathbf{M}_{n+1}}\left[\mathrm{E}\left(F \mid \mathbf{D}, \mathbf{M}_{n+1}\right)\right]$,

179 where $\mathrm{E}_{\mathbf{M}_{n+1}}\left[\operatorname{Var}\left(F \mid \mathbf{D}, \mathbf{M}_{n+1}\right)\right]$ is the within-model variance of fluoride concentration prediction

180 using models at level $n+1$ calculated by

181

$$
\mathrm{E}_{\mathbf{M}_{n+1}}\left[\operatorname{Var}\left(F \mid \mathbf{D}, \mathbf{M}_{n+1}\right)\right]=\sum_{q} \operatorname{Var}\left(F \mid \mathbf{D}, \mathbf{M}_{n+1}^{(q)}\right) \operatorname{Pr}\left(\mathbf{M}_{n+1}^{(q)} \mid \mathbf{D}, \mathbf{M}_{n}\right)
$$

$182 \operatorname{Var}_{\mathbf{M}_{n+1}}\left[\mathrm{E}\left(F \mid \mathbf{D}, \mathbf{M}_{n+1}\right)\right]$ is the between-model variance using models at level $n+1$ calculated by

$$
\begin{aligned}
\operatorname{Var}_{\mathbf{M}_{n+1}}\left[\mathrm{E}\left(F \mid \mathbf{D}, \mathbf{M}_{n+1}\right)\right] & =\mathrm{E}_{\mathbf{M}_{n+1}}\left[\left(\mathrm{E}\left(F \mid \mathbf{D}, \mathbf{M}_{n+1}\right)-\mathrm{E}\left(F \mid \mathbf{D}, \mathbf{M}_{n}\right)\right)^{2}\right] \\
& =\sum_{q}\left[\mathrm{E}\left(F \mid \mathbf{D}, \mathbf{M}_{n+1}^{(q)}\right)-\mathrm{E}\left(F \mid \mathbf{D}, \mathbf{M}_{n}\right)\right]^{2} \operatorname{Pr}\left(\mathbf{M}_{n+1}^{(q)} \mid \mathbf{D}, \mathbf{M}_{n}\right)
\end{aligned}
$$

$184 \operatorname{Var}_{\mathbf{M}_{n+1}}$ is the variance operator with respect to models $\mathbf{M}_{n+1}$.

186 model $\mathbf{M}_{n}$ at level $n$ is (Chitsazan and Tsai, 2015a,b)

$$
\operatorname{Pr}\left(\mathbf{M}_{n+1}^{(q)} \mid \mathbf{D}, \mathbf{M}_{n}\right)=\frac{\operatorname{Pr}\left(\mathbf{M}_{n+1}^{(q)} \mid \mathbf{D}\right)}{\operatorname{Pr}\left(\mathbf{M}_{n} \mid \mathbf{D}\right)}
$$


model of its parent model $\mathbf{M}_{n}, \operatorname{Pr}\left(\mathbf{M}_{n+1}^{(q)} \mid \mathbf{D}\right)$ also represents the joint probability of $\mathbf{M}_{n+1}^{(q)}$ and

$190 \mathbf{M}_{n} \cdot \operatorname{Pr}\left(\mathbf{M}_{n} \mid \mathbf{D}\right)$ is the posterior model probability for model $\mathbf{M}_{n} \cdot \operatorname{Pr}\left(\mathbf{M}_{n} \mid \mathbf{D}\right)$ is calculated by

$191 \operatorname{Pr}\left(\mathbf{M}_{n} \mid \mathbf{D}\right)=\sum_{q} \operatorname{Pr}\left(\mathbf{M}_{n+1}^{(q)} \mid \mathbf{D}\right)$

\subsection{Between Model Uncertainty Analysis for Two Sources of Model Structure Uncertainty}

This study considers two sources of model structure uncertainty in two level as between

194 model uncertainty. The first level source of uncertainty is from the choice of an activation

195 function for the hidden layer. A BMA model at level 1 is denoted as $\mathbf{M}_{1}^{(p)}$. The second level

196 considers the source of uncertainty from the choice of the number of hidden layer nodes. Under

197 an activation function, an ANN model at level 2 (base level) is denoted as $\mathbf{M}_{2}^{(q)}$, which is a child

198 model of $\mathbf{M}_{1}^{(p)}$. We denote the set of ANN models as $\mathbf{M}_{2}^{(q)}=\left\{A N N_{p, q}\right\}$.

Based on Bayes' theorem (Shafer, 1976), the posterior model probability of an ANN model given an activation function and a number of hidden layer nodes is

$$
\operatorname{Pr}\left(A N N_{p, q} \mid \mathbf{D}\right)=\operatorname{Pr}\left(\mathbf{M}_{2}^{(q)} \mid \mathbf{D}\right)=\frac{\operatorname{Pr}\left(\mathbf{D} \mid \mathbf{M}_{1}^{(p)}, \mathbf{M}_{2}^{(q)}\right) \times \operatorname{Pr}\left(\mathbf{M}_{2}^{(q)} \mid \mathbf{M}_{1}^{(p)}\right) \times \operatorname{Pr}\left(\mathbf{M}_{1}^{(p)}\right)}{\sum_{p} \sum_{q} \operatorname{Pr}\left(\mathbf{D} \mid \mathbf{M}_{1}^{(p)}, \mathbf{M}_{2}^{(q)}\right) \times \operatorname{Pr}\left(\mathbf{M}_{2}^{(q)} \mid \mathbf{M}_{1}^{(p)}\right) \times \operatorname{Pr}\left(\mathbf{M}_{1}^{(p)}\right)}
$$

202 where $\operatorname{Pr}\left(\mathbf{D} \mid \mathbf{M}_{1}^{(p)}, \mathbf{M}_{2}^{(q)}\right)$ is the marginal likelihood function of a base model given an

203 activation function and a number of hidden layer nodes, $\operatorname{Pr}\left(\mathbf{M}_{2}^{(q)} \mid \mathbf{M}_{1}^{(p)}\right)$ is the conditional prior

204 probability of a base model that uses a number of hidden layer nodes given an activation

205 function. And $\operatorname{Pr}\left(\mathbf{M}_{1}^{(p)}\right)$ is the prior probability of a BMA model that uses an activation 206 function. 
208 an activation function and all proposed numbers of hidden layer nodes is

$209 \operatorname{Pr}\left(\mathbf{M}_{1} \mid \mathbf{D}\right)=\sum_{q} \operatorname{Pr}\left(\mathbf{M}_{2}^{(q)} \mid \mathbf{D}\right)$

210 The conditional posterior probability of an ANN model that uses a proposed number of hidden

211 layer nodes given an activation function is

$212 \operatorname{Pr}\left(\mathbf{M}_{2}^{(q)} \mid \mathbf{M}_{1}, \mathbf{D}\right)=\frac{\operatorname{Pr}\left(\mathbf{M}_{2}^{(q)} \mid \mathbf{D}\right)}{\operatorname{Pr}\left(\mathbf{M}_{1} \mid \mathbf{D}\right)}$

213 Using the Laplace approximation (Tierney and Kadane, 1986), the marginal likelihood function

$214 \operatorname{Pr}\left(\mathbf{D} \mid \mathbf{M}_{2}^{(q)}\right)$ can be approximated by the Bayesian information criterion (BIC) (Li and Tsai,

215 2009) as

$216 \operatorname{Pr}\left(\mathbf{D} \mid \mathbf{M}_{1}^{(p)}, \mathbf{M}_{2}^{(q)}\right) \approx \exp \left[-\frac{1}{2} \mathrm{BIC}_{p, q}\right]$

217 where

$218 \quad \mathrm{BIC}_{p, q}=Q_{p, q}\left(\boldsymbol{\beta}_{p, q}\right)+n \ln 2 \pi+m_{p, q} \ln n$

219 where $Q_{p, q}$ is the sum of the weighted square errors, $\boldsymbol{\beta}_{p, q}$ is the maximum-likelihood estimated 220 parameters (weights and biases) for an ANN model. $\mathrm{n}$ is the size of "in-sample" data, and $m_{p, q}$ 221 is the size of $\boldsymbol{\beta}_{p, q}$.

222 This study assumes equal prior probability for $\operatorname{Pr}\left(\mathbf{M}_{1}^{(p)}\right)$ and equal conditional prior 223 probability for $\operatorname{Pr}\left(\mathbf{M}_{2}^{(q)} \mid \mathbf{M}_{1}^{(p)}\right)$. By substituting equation (14) to equation (11) and using the 224 variance window (Tsai and $\mathrm{Li}, 2008 \mathrm{a}$,b; Singh et al., 2010) to enlarge the acceptance window 225 size, the ANN posterior model probabilities can be obtained as follows 
$226 \operatorname{Pr}\left(A N N_{p, q} \mid \mathbf{D}\right)=\operatorname{Pr}\left(\mathbf{M}_{2}^{(q)} \mid \mathbf{D}\right)=\frac{\exp \left[-\frac{1}{2} \alpha \Delta \mathrm{BIC}_{p, q}\right]}{\sum_{p} \sum_{q} \exp \left[-\frac{1}{2} \alpha \Delta \mathrm{BIC}_{p, q}\right]}$

227 where $\Delta \mathrm{BIC}_{p, q}=\mathrm{BIC}_{p, q}-\mathrm{BIC}_{\min }$. $\mathrm{BIC}_{\min }$ is the smallest BIC value among the ANN models. $\alpha$

228 is the scaling. The readers are referred to Tsai and $\operatorname{Li}$ (2008a) for choosing an $\alpha$ value.

2292.4 Fluoride Concentration Prediction and Uncertainty by HBMA

230 Using equation (4), fluoride concentration prediction by the hierarch model, which uses

231 all of the propositions of the activation functions and the numbers of hidden layers, is

$232 \mathrm{E}(F \mid \mathbf{D})=\mathrm{E}_{\mathbf{M}_{1}} \mathrm{E}_{\mathbf{M}_{2}}\left[\mathrm{E}\left(F \mid \mathbf{D}, \mathbf{M}_{2}\right)\right]$

233 where $\mathrm{E}\left(F \mid \mathbf{D}, \mathbf{M}_{2}\right)$ is the mean fluoride concentration predicted by an ANN model at the base

234 level. The mean fluoride concentration predicted by BMA at level 1 is

$235 \mathrm{E}\left(F \mid \mathbf{D}, \mathbf{M}_{1}^{(p)}\right)=\mathrm{E}_{\mathbf{M}_{2}}\left[\mathrm{E}\left(F \mid \mathbf{D}, \mathbf{M}_{2}\right)\right]$

236 The total variance of the fluoride prediction using the hierarch model is

$\operatorname{Var}(F \mid \mathbf{D})=\mathrm{E}_{\mathbf{M}_{1}} \mathrm{E}_{\mathbf{M}_{2}} \operatorname{Var}\left(F \mid \mathbf{D}, \mathbf{M}_{2}\right)+\mathrm{E}_{\mathbf{M}_{1}} \operatorname{Var}_{\mathbf{M}_{2}} \mathrm{E}\left(F \mid \mathbf{D}, \mathbf{M}_{2}\right)+\operatorname{Var}_{\mathbf{M}_{1}} \mathrm{E}_{\mathbf{M}_{2}} \mathrm{E}\left(F \mid \mathbf{D}, \mathbf{M}_{2}\right)$

238 where $\operatorname{Var}\left(F \mid \mathbf{D}, \mathbf{M}_{2}\right)$ is the variance of predicted fluoride concentration by an ANN model at 239 the base level.

240 The total variance consists of three variance terms. The first term in equation (19) is the

241 within-model variance of the prediction, which stems from the uncertain inputs and uncertain

242 parameters. The second and the third terms in equation (19) stem from the uncertainty in the

243 number of hidden layer nodes and the uncertainty in the hidden layer activation function, 244 respectively.

245 The variance of fluoride concentration prediction using a BMA model at level 1 is 
$246 \operatorname{Var}\left(F \mid \mathbf{D}, \mathbf{M}_{1}\right)=\mathrm{E}_{\mathbf{M}_{2}}\left[\operatorname{Var}\left(F \mid \mathbf{D}, \mathbf{M}_{2}\right)\right]+\operatorname{Var}_{\mathbf{M}_{2}}\left[\mathrm{E}\left(F \mid \mathbf{D}, \mathbf{M}_{2}\right)\right]$

247 Equations (19)-(20) enable us to explicitly evaluate the contribution of individual sources of 248 uncertainty in the total prediction variance.

$249 \quad 2.5$ Within-Model Variance Derivation

250 Given an ANN structure, the within-model variance of prediction $\operatorname{Var}\left(F \mid \mathbf{D}, \mathbf{M}_{2}\right)$ can be

251 estimated through Monte Carlo (MC) simulation as long as the variance of the ANN inputs and

252 ANN parameters are known. In this study, the variance of the input data at unsampled location is

253 estimated by the kriging variance that stems from using the kriging algorithm to interpolate the

254 input data to unsampled location. To quantify the variance of ANN parameters, we follow Yeh

255 and Yoon $(1976,1981)$ that the covariance matrix of estimated model parameters $\boldsymbol{\beta}_{p, q}$ is

$256 \operatorname{Cov}_{F}\left(\boldsymbol{\beta}_{p, q}\right)=\hat{\sigma}_{F}^{2}\left(J_{F}^{T} J_{F}\right)$

257 where $\hat{\sigma}_{F}^{2}$ is the estimated variance of fluoride concentration, which is

$258 \hat{\sigma}_{F}^{2}=\frac{\sum_{k=1}^{n}\left[F_{k}\left(A N N_{p, q}, \boldsymbol{\beta}_{p, q}\right)-F_{k}^{o b s}\right]^{2}}{n-m_{p, q}}$

259 where $F_{k}\left(A N N_{p, q}, \boldsymbol{\beta}_{p, q}\right)$ is the predicted fluoride concentration by $A N N_{p, q}$ using the model

260 parameters $\boldsymbol{\beta}_{p, q}$ and $F_{k}^{o b s}$ is the measured fluoride concentration. $J_{F}=\partial F / \partial \hat{\boldsymbol{\beta}}_{p, q}$ is the Jacobian

261 matrix of the fluoride concentration to model parameters, which is

$262 \quad J_{F}=\left[\begin{array}{llll}\frac{\partial F}{\partial W_{i, j}} & \frac{\partial F}{\partial b_{j}} & \frac{\partial F}{\partial W_{j}} & \frac{\partial F}{\partial b}\end{array}\right]$

263 According to equations (1) and (2), the derivatives of fluoride concentration $F_{k}$ to the ANN 264 weights $W_{i, j}$ and biases $b_{j}$ are 
$265 \quad \frac{\partial F_{k}}{\partial W_{i j}}=\frac{\partial f_{1}\left(L_{j k}\right)}{\partial L_{j k}} W_{j} I_{i k}$

$266 \frac{\partial F_{k}}{\partial b_{j}}=\frac{\partial f_{1}\left(L_{j k}\right)}{\partial L_{j k}} W_{j}$

267 where $L_{j k}=b_{j}+\sum_{i} W_{i j} I_{i k}$. The derivatives of fluoride concentration $F_{k}$ to the ANN weights $W_{j}$ 268 and biases $b$ are

$269 \frac{\partial F_{k}}{\partial W_{j}}=f_{1}\left(L_{j k}\right)$

$270 \quad \frac{\partial F_{k}}{\partial b}=1$

271 Using the covariance matrix of estimated parameters in equation (23) and the kriging 272 variances of the input data, Monte Carlo simulation was conducted to determine the within273 model variance of the fluoride concentration for each ANN model.

\section{3. Study Area}

The Maku area shown in Figure 2 is located in the West Azarbaijan province, northwest

276 of Iran. The total study area is about $1595 \mathrm{Km}^{2}$, where up to $397 \mathrm{Km}^{2}$ is covered by basaltic

277 lavas bounded on the west by Turkey and on the east by Aras River. The main residential areas

278 in the region are Maku and Poldasht. The climate in the area is cold and arid with annual-mean

279 temperature $11.2^{\circ} \mathrm{C}(1985-2012)$. The annual-mean precipitation is about $286.6 \mathrm{~mm}$ (1992-

280 2012). The maximum and minimum precipitations occur in May and September, respectively.

281 Total evaporation is $1500 \mathrm{~mm}$ per year, three times more than annual precipitations (Asghari

282 Moghaddam and Fijani, 2008). The main rivers in the study area are Sari Su and Zangmar, which

283 flow from west to east. The main source of water is groundwater to meet the demands for

284 different purposes such as drinking, agriculture and industry. Groundwater discharges at 12 
285 large-scale springs ranging from 20 to $4000 \mathrm{Ls}^{-1}$ and at several withdrawal wells (Asghari 286 Moghaddam and Fijani 2009).

287 The study area is covered by two major geological formations: (a) the basaltic area, 288 where the aquifer mainly consists of basaltic rocks and covered by alluvium (basalt-alluvium 289 aquifer), and (b) the non-basaltic area, where the aquifer consists of limestones of Qom 290 formation (Oligo-Miocene age) and massive limestone and dolomites of Ruteh formation 291 (Permian). Other formations, such as shale, marl and conglomerate, are present in small areas. 292 Prior studies (Asghari Moghaddam and Fijani 2008) showed that ion concentration, electrical 293 conductivity (EC), total dissolved solid (TDS) and alkalinity in the basaltic aquifer are higher 294 than those in the non-basaltic aquifer and in the mixing zones where non-basaltic water and 295 basaltic water mix (Asghari Moghaddam and Fijani 2009).

\section{4. Data Analysis}

297 To estimate the fluoride concentration in the study area, 129 water samples were 298 collected over water sources (wells, springs, ganats, etc.) in a five-year period 2004-2008. The 299 sample locations are shown in Figure 2. The samples were analyzed in a hydrogeological 300 laboratory in the University of Tabriz using the standard procedure of APHA (1998) for fluoride 301 and other 11 hydrochemical parameters. The observed precision of ion analysis was within the 302 range of acceptability $( \pm 5 \%)$ used in most of chemical analysis. After the correlation matrix and 303 factor analyses (Nadiri et al., 2013), four highly correlated parameters $\left(\mathrm{Na}^{+}, \mathrm{K}^{+}, \mathrm{HCO}_{3}^{-}\right.$, and $304 \mathrm{Ca}^{2+}$ ) to fluoride concentration are chosen as input data for the ANN models. 110 samples were 305 used in the training step ("in-sample" data set) and 19 samples were used in the test step ("out306 sample" data set). The training and test data sets have similar statistical characteristics. Different 
307 types of the input data were scaled to have a range between 0 and 1 and the samples were

308 selected to cover the entire area.

\section{5. Results and Discussion}

\section{$310 \quad 5.1$ Model Selection}

For simplicity, we use the activation function name followed by the number of hidden

312 layer nodes to denote an ANN model. For example "Tansig-6" represents an ANN model that

313 uses the Tansig activation function and six nodes in the hidden layer. Figure 3 shows the BIC

314 values and posterior model probabilities for the 16 trained ANNs. From left to right, the first

315 eight super columns represent the ANNs using Logsig activation function. The second eight

316 super columns represent ANNs using Tansig activation function. Each super column in Figure 3

317 consists of two sub-columns showing the weighted sum of squared errors $\left(Q_{p, q}\right)$ and the BIC

318 complexity term $\left(N \ln 2 \pi+m_{p} \ln N\right)$. By increasing the number of nodes in the hidden layer, the

319 complexity of the ANNs increases, which decreases the $Q_{p}$ value, but risks the increase in BIC

320 value. For example, given the Tansig activation function, BIC value decreases by increasing the

321 number of the hidden layer nodes from two to six. However, BIC starts to increase for more than

322 six nodes because the reduction in $Q_{p}$ is not sufficient to compensate the increase of the

323 complexity term. From the model selection perspective, the best neural network is Tansig- 6 with

324 the minimum $\mathrm{BIC}=447.33$. The coefficient of determination $\left(R^{2}\right)$ and the root mean squared

325 errors (RMSE) of Tansig- 6 model are $R^{2}=0.9608$ and RMSE $=0.0413$ for the "in-sample" data

326 and $R^{2}=0.4946$ and $\mathrm{RMSE}=0.0582$ for the "out-sample" data, respectively. This result indicates

327 that although BIC is a sound criterion for model selection, the best model does not perform well

328 for the "out-sample" data. In the following discussion, we apply the HBMA to address this 329 problem. 


\subsection{HBMA Results}

Figure 3 shows the posterior model probabilities for the ANN models using Occam's

332 window $(\alpha=1)$ and variance window ( $\alpha=0.404,0.202$ and 0.101$)$ with $5 \%$ significant level

333 and two, three and four standard deviations of errors. Occam's window only accepts the best

334 model "Tansig-6" and the second best model "Logsig-4" with $92.56 \%$ and $7.44 \%$ posterior

335 model probabilities, respectively. Tsai and Li, (2008a, b) noted that when the number of

336 observation data increases, Occam's window tends to single out the best model. By introducing

337 the scaling factor that considers the factor of data size, variance window includes more models as

338 shown in Figure 3 for analysis.

339 Figure 4 shows the comparisons of the "out-sample" data against the mean fluoride

340 concentration predicted by the hierarch model given Occam's window and thee variance window

341 sizes. Using Occam's window, $R^{2}$ and RMSE are 0.5593 and 0.0543 , respectively, which

342 performs better than Tansig-6 model. However, the improvement is smaller than those using

343 variance windows because Occam's window only accepts the best two ANN models. The 4- $\sigma$

344 variance window size has the highest $R^{2}=0.7862$ and lowest $\mathrm{RMSE}=0.0378$. This result

345 supports the Ahmad and Zhang, (2002) conclusion that combining the neural networks improves

346 the robustness of predictions by sharing and averaging the prediction errors. In this study, we

347 choose $4 \sigma$ variance window with $5 \%$ significance level for further analysis.

348 Figure 5 shows the BMA tree of conditional posterior model probabilities and posterior

349 model probabilities. Tansig-6 and Logsig-4 at level 2 are the best ANN model and the second

350 best ANN model with $24.20 \%$ and $18.67 \%$ posterior model probabilities, respectively. Tansig

351 and Logsig models at level 1 have similar conditional model posterior probability close to 50\%,

352 which indicates that both activation functions are equally important in producing the outcome. 
353 Given the Logsig activation function, the ANN model with four nodes in the hidden layer has the

354 highest conditional posterior model probability $36.51 \%$ followed by the model with three nodes

355 in the hidden layer with $28.69 \%$ posterior model probability. However, given the Tansig

356 activation function, the ANN models with six and seven nodes in the hidden layer are the best

357 and second best models with $49.53 \%$ and $18.68 \%$ posterior model probability, respectively. This

358 result indicates that the optimal number of hidden layer nodes for an ANN model depends on the

359 hidden layer activation function.

\section{$360 \quad 5.3$ Comparison between Ensemble Methods}

361 In this section, we compare the performance of the best neural network (Tansig-6), the

362 hierarch model, the committee machines (CM) (Perrone and Cooper, 1993; Wong et al., 2000;

363 Chen and Lin, 2006; Kadkhodaie-Ilkhchi et al. 2009; Labani et al. 2010), and the supervised

364 committee machines (SCM) (Drucker, 1997; Hu and Tsoukalas, 2003; Nadiri et al., 2013) for

365 fluoride concentration prediction. Table 1 summarizes the formulation of the compared ANN

366 ensemble methods. The CM outputs are the simple averaged or the weighted averaged of the

367 predictions of individual ANNs. The SCM outputs are the nonlinear combination of the

368 predictions of individual ANNs, provided by a supervised ANN model. In other words, SCM

369 receives the predictions of individual ANNs as input, and provides new predictions with reduced

370 fitting error to the "in-sample" data set.

371 Table 2 lists the maximum error, RMSE and the $R^{2}$ of the estimated fluoride

372 concentrations for the "in-sample" and "out-sample" data sets. All ensemble methods show less

373 errors and better fitting than the best model Tansig-6. This demonstrates the potential risk of

374 using a single ANN model and the importance of using multiple models for prediction. Ensemble 
375 methods significantly reduce the maximum error by averaging the errors from different neural 376 networks.

377 For the "in-sample" data set, the supervised committee machines has the best fitting 378 error, followed by the weighted averaging committee machines, the hierarch model, and the 379 simple averaging committee machine. However, the supervised committee machines and the 380 weighted averaging committee machines performed poor to the "out-sample" data set because 381 both methods do not consider the complexity of the ANN models in combining the predictions of 382 ANN models. The hierarch model has the best fitting to the "out-sample" data set. Also, the 383 simple averaging committee machine performs better than SCM and CM for the "out-sample"

384 data set. This draws an important remark that methods with less fitting errors for the "in-sample" 385 data set do not necessarily have better performance for the "out-sample" data set. The parsimony 386 principle should be taken into account to sensibly determine the combination of ANN 387 predictions.

Moreover, using the weighted averaging committee machine, the ANNs with less than six

389 nodes in the hidden layer are virtually ignored. However, the posterior model probabilities in 390 Figure 5 suggest models with less than six nodes in the hidden layer can be important. The result 391 supports the HBMA method since the posterior model probabilities are more meaningful than 392 either the optimized model weights or equal model weights in the committee machines.

\section{$393 \quad 5.4$ Fluoride Estimation Variances}

394 Figure 6 shows the prediction variances for the "out-sample" data set. Each super-column 395 consists of three sub-columns from bottom to top showing the variances from uncertainty in 396 ANN weights and biases (within-model variance), the variances from uncertainty in the number 
397 of hidden layer nodes and the variances from uncertainty in the hidden layer activation function.

398 The sum of the second and third sub-columns shows the between-model variance.

To calculate the within-model variance of each ANN model, we used the equations (21)-

400 (27) to calculate the variance of the neural network weights and biases. Then, realizations of the

401 weights and biases are generated by Monte-Carlo simulation and are used in the neural network

402 to generate ensemble variance of fluoride concentration.

403 We use the result in Figure 6 to calculate the variance contributions of individual sources

404 of uncertainty to the total prediction variance. The within-model variance contributes from

$40598.24 \%$ to $54.09 \%$ to the total variances and is the most important source of uncertainty. It

406 indicates that reducing uncertainty in the ANN parameters is more important than ANN

407 structures. The hidden layer node uncertainty contributes from $43.19 \%$ to $1.74 \%$ and the

408 activation function uncertainty contributes $18.03 \%$ to $0.01 \%$ to the total variances. The low

409 variance contribution in the activation function shows that the neural networks with different

410 activation functions can have similar performance. In other words, given an "in-sample" data set,

411 for different activation functions, neural networks adjust their parameters in a way that lead to

412 similar performance. The total between model variance contributes from $45.90 \%$ to $1.76 \%$,

413 respectively. This result indicates that although the within-model variances are more important

414 the between-model variances are also significant and cannot be ignored.

415 Figure 7 shows, using the best neural network (Tansig-6), three out of the 19 "out-

416 sample" fluoride measurements has higher than one standard deviation estimation error.

417 However, using the hierarch model, all of 19 measurements have less than one standard

418 deviation error. The reason is that the best neural network underestimates the prediction

419 variances by ignoring the between-model variances. 


\subsection{Spatial Fluoride Prediction and Prediction Variances in Maku Area}

Figure 8(a) shows the estimated mean fluoride concentration map for the Maku area by

422 the hierarch model. The fluoride concentration is high in the south area between the Maku and

423 Poldasht rivers, where the aquifer is mainly consist of basaltic rocks and covered by alluvium

424 (basalt-alluvium aquifer). This result shows a significant correlation between the basaltic

425 formation and the presence of fluoride in the aquifer system. Figure 8(b) shows the standard 426 deviation map of the estimated fluoride concentration that stems from the kriging variance of the

427 input data, the variance of the ANN model parameters and the uncertainty of ANN model

428 structures. It is observed that the estimation standard deviations are less in the basaltic area. This

429 observation shows that different ANN models predict similar high fluoride concentration in the

430 basaltic area.

\section{6. Conclusion}

433 This study introduces the hierarchical Bayesian model averaging (HBMA) to combine the 434 predictions of multiple artificial neural networks (ANNs) and assess the uncertainty of the 435 predictions. The HBMA is able to segregate the variances from different sources in order to 436 explicitly evaluate the contribution of each source to the total uncertainty. The HBMA prediction 437 results are found better than using a single ANN model.

438 This study shows that combining the ANN predictions can reduce the prediction errors by 439 averaging the errors of individual models. The results also illustrate that the ensemble methods 440 with less fitting errors for the "in-sample" data set do not necessarily have better performance for 441 the "out-sample" data set. This study found that the BMA outperforms the committee machines 
442 because BMA considers the parsimony principle in determining the posterior model 443 probabilities.

444 The prediction variances are also quantified by using the HBMA approach. It was found 445 that the high predicted fluoride concentration in the basaltic area is attributed to small variance, 446 which shows strong correlation between the basaltic formations and the presence of fluoride in

447 the groundwater system. In addition, by segregating the sources of uncertainty HBMA can 448 calculate the contribution of each sources of uncertainty in the total prediction variance. The 449 results show that the variances from ANN uncertain inputs and parameters are the most 450 important prediction variances followed by the prediction variances from uncertain number of 451 hidden layer nodes and uncertain activation function. The contribution of between-model 452 variance in the total prediction variance is smaller than the within-model variances. However, 453 they are in the same order of magnitude; therefore, ignoring the between-model variances might 454 lead to underestimating the prediction variances.

\section{Acknowledgements}

457 This study was supported by the Grant/Cooperative Agreement Number G10AP00136 458 from the United States Geological Survey. The authors acknowledge Elham Fijani and Asghar 459 Asghari Moghaddam for providing hydrochemical data of the Maku area for this study.

\section{References}

462 Ablameyko, S., Goras, L., Gori, M., \& Piuri, V., 2003. Neural networks for instrumentation, measurement and related industrial applications. NATO Science Series: Computer\& Systems Sciences 185. The Netherlands: IOS Press.

Ahmad, Z., and J. Zhang, 2002. A comparison of different methods for combining multiple neural networks models, in proceedings of the 2002 World Congress on Computational Intelligence, pp. 12- 117, IEEE Press, Piscataway, N.J. 
Anderson, D., McNeill, G., 1992. Artificial neural networks technology. ADACS State-of-theArt Report, Contract Number F30602-89-C-0082, Rome Laboratory, Kaman Sciences Corporation. New York 13502-4627.

Asghari Moghaddam, A. \& Fijani, E., 2008. Distribution of fluoride in groundwater of Maku area, northwest of Iran. Environmental Geology 56(2), 281-287.

Asghari Moghaddam, A. \& E. Fijani, 2009. Hydrogeologic framework of the Maku area basalts, northwestern Iran. Hydrogeology Journal 17(4), 949-959.

Bishop, C.M., 1995. Neural networks for pattern recognition. Oxford university press.

Box, G. E. P., 1976. Science and statistics. Journal of the American Statistical Association, 71(356), 791-799.

Chen, C.-H., and Z.-S. Lin, 2006. A committee machine with empirical formulas for permeability prediction. Computers \& geosciences, 32(4), 485-496.

Chitsazan, N. and F.T.-C. Tsai. 2015a. Uncertainty segregation and comparative evaluation in groundwater remediation designs: A chance-constrained hierarchical Bayesian model averaging approach, Journal of Water Resources Planning and Management, 141(3), 04014061. doi:10.1061/(ASCE)WR.1943-5452.0000461

Chitsazan, N. and F.T.-C. Tsai. 2015b. A hierarchical Bayesian model averaging framework for groundwater prediction under uncertainty, Groundwater, 53(2), 305-316. doi:10.1111/gwat.12207

Coppola, E., Rana, A.J., Poulton, M.M., Szidarovszky, F., Uhl, V.V., 2005. A neural network model for predicting aquifer water level elevations. Ground Water 43, 231-241.

Cybenko, G., 1989. Approximations by superpositions of a sigmoidal function, Mathematics of Control Signals and Systems. 2, 303-314

Daliakopoulos, I.N., P. Coulibaly, and I.K. Tsanis, 2005. Groundwater level forecasting using artificial neural networks. Journal of Hydrology, 309(1), 229-240.

Dawson, C., and R. Wilby, 2001. Hydrological modeling using artificial neural networks. Progress in physical Geography, 25(1), 80-108.

Draper, D., 1995. Assessment and propagation of model uncertainty. Journal of the Royal Statistical Society. Series B (Methodological), 45-97.

Drucker, H., 1997. Improving regressors using boosting techniques, in proceedings of the 14th international conference on machine learning, pp. 107-115.

Franses, P.H., and G. Draisma, 1997. Recognizing changing seasonal patterns using artificial neural networks. Journal of Econometrics 81(1), 273-280.

Geyer, C. J., 1992. Practical Markov chain Monte Carlo. Statistical Science, 7(4), 473-483.

Govindaraju, R.S., 2000a. Artificial neural networks in hydrology. I: Preliminary concepts. Journal of Hydrologic Engineering 5(2), 115-123.

Govindaraju, R.S., 2000b. Artificial neural networks in hydrology. II: hydrologic applications. Journal of Hydrologic Engineering 5(2), 124-137.

Haario, Heikki, Eero Saksman, and Johanna Tamminen, 2001. An adaptive Metropolis algorithm. Bernoulli 7(2), 223-242.

Hoeting, J. A., D. Madigan, A. E. Raftery, and C. T. Volinsky, 1999. Bayesian model averaging: A tutorial. Statistical science 14(4), 382- 401.

Hsu, K., Gupta, H.V., Sorooshian, S., 1995. Artificial neural network modeling of the rainfallrunoff process. Water Resource. Research. 31(10), 2517-2530. 
Hu, M.Y., and C. Tsoukalas, 2003. Explaining consumer choice through neural networks: The stacked generalization approach. European Journal of Operational Research 146(3), 650660.

Jha, M. K., and S. Sahoo, 2015. Efficacy of neural network and genetic algorithm techniques in simulating spatio-temporal fluctuations of groundwater. Hydrological Processes, 29(5), 671-691.

Kadkhodaie-Ilkhchi, A., Rahimpour-Bonab, H. \& Rezaee, M, 2009. A committee machine with intelligent systems for estimation of total organic carbon content from petrophysical data: an example from Kangan and Dalan reservoirs in South Pars Gas Field, Iran. Computational Geosciences. 35 (3), 459-474.

Khan, M.S., and P. Coulibaly, 2006. Bayesian neural network for rainfall-runoff modeling. Water resources research 42(7), W07409.

Kingston, G.B., M.F. Lambert, and H.R. Maier, 2005. Bayesian training of artificial neural networks used for water resources modeling. Water resources research 41(12), W12409.

Kingston, G. B., H. R. Maier, and M. F. Lambert, 2008. Bayesian model selection applied to artificial neural networks used for water resources modeling. Water resources research 44(4), W04419.

Kumar, A. R. S., Sudheer, K. P., Jain S. K., P. K. Agarwal, 2005. Rainfall-runoff modelling using artificial neural networks: comparison of network types. Hydrological Processes 19(6), 1277-1291.

Labani, M. M., Kadkhodaie-Ilkhchi, A. and Salahshoor, K, 2010. Estimation of NMR $\log$ parameters from conventional well log data using a committee machine with intelligent systems: a case study from the Iranian part of the South Pars gas field, Persian Gulf Basin. Journal of Petroleum Science and Engineering. 72 (1-2), 175-185.

Li, X., Tsai, F.T.-C., 2009. Bayesian model averaging for groundwater head prediction and uncertainty analysis using multimodel and multimethod. Water Resource Research 45(9), W09403.

Maier, H.R., and G.C. Dandy, 2000. Neural networks for the prediction and forecasting of water resources variables: a review of modeling issues and applications. Environmental Modelling \& Software 15(1), 101-124.

Müller, P., and D.R. Insua, 1998. Issues in Bayesian analysis of neural network models. Neural Computation 10(3), 749-770.

Nadiri, A.A., Fijani, E., Tsai, F. T.-C., Asghari Moghaddam, A., 2013. Supervised committee machine with artificial intelligence for prediction of fluoride concentration. Journal of Hydroinformatics 15(4), 1474-1490.

Nadiri, A.A., N. Chitsazan, F.T.-C. Tsai, and A.A. Moghaddam, 2014. Bayesian artificial intelligence model averaging for hydraulic conductivity estimation. Journal of Hydrologic Engineering 19(3), 520-532.

Neal, R. M., 1995. Bayesian learning for neural networks. Doctoral dissertation, University of Toronto.

Nourani, V., Mogaddam, A. A., Nadiri, A. O., Singh, V. P., 2008a. Forecasting spatiotemporal water levels of Tabriz aquifer. Trends in Applied Sciences Research 3(4), 319-329.

Nourani, V., Mogaddam, A. A., Nadiri, A. O., 2008b. An ANN-based model for spatiotemporal groundwater level forecasting. Hydrological Processes 22(26), 5054-5066. 
Perrone, M. P., and L. N. Cooper 1993. When networks disagree: Ensemble methods for hybrid neural networks, in Artificial Neural Networks for Speech and Vision, edited by R. J. Mammone, pp. 126-142, Chapman and Hall, New York.

Qi, M., Zhang, G.P., 2001. An investigation of model selection criteria for neural network time series forecasting. European Journal of Operational Research 132, 666-680

Rogers, L.L., and F.U. Dowla, 1994. Optimization of groundwater remediation using artificial neural networks with parallel solute transport modeling. Water resources research 30(2), 457-481.

Sahoo, G.B., Ray, C., 2006. Flow forecasting for a Hawaii stream using rating curves and neural networks. Journal of Hydrology 317, 63-80.

Sahoo, G.B., C. Ray, E. Mehnert, and D.A. Keefer, 2006. Application of artificial neural networks to assess pesticide contamination in shallow groundwater. Science of the total environment 367(1), 234-251.

Shafer, G., 1976. A mathematical theory of evidence, Princeton University press Princeton.

Sharma, V., S. Negi, R. Rudra, and S. Yang, 2003. Neural networks for predicting nitratenitrogen in drainage water. Agricultural water management, 63(3), 169-183.

Shu, C., and D.H. Burn, 2004. Artificial neural network ensembles and their application in pooled flood frequency analysis. Water resources research 40(9), W09301.

Singh, A., S. Mishra, and G. Ruskauff, 2010. Model averaging techniques for quantifying conceptual model uncertainty. Ground Water 48(5), 701-715.

Stone, C.J., 1981. Admissible selection of an accurate and parsimonious normal linear regression model. The Annals of Statistics 9(3), 475-485.

Sudheer, K. P., A. Gosain, D. Mohana Rangan, and S. Saheb, 2002. Modelling evaporation using an artificial neural network algorithm. Hydrological processes 16(16), 3189-3202.

Sudheer, K. P., P. C. Nayak and K. S. Saheb, 2003. Improving peak flow estimates in artificial neural network river flow models. Hydrological Processes 17(3), 677-686.

Tetko, I.V., D.J. Livingstone, and A.I. Luik, 1995. Neural network studies. 1. Comparison of overfitting and overtraining. Journal of chemical information and computer sciences 35(5), 826-833.

Tierney, L., and J.B. Kadane, 1986. Accurate approximations for posterior moments and marginal densities. Journal of the American Statistical Association, 81(393), 82-86.

Tsai, F.T.-C., and X. Li, 2008a. Inverse groundwater modeling for hydraulic conductivity estimation using Bayesian model averaging and variance window. Water Resources Research 44(9), W09434.

Tsai, F.T.-C., and X. Li, 2008b. Multiple parameterization for hydraulic conductivity identification. Ground Water, 46(6), 851-864.

Tsai, F.T.-C., and A.S. Elshall. 2013. Hierarchical Bayesian model averaging for hydrostratigraphic modeling: Uncertainty segregation and comparative evaluation. Water Resources Research, 49, 5520-5536.

Valverde Ramírez, M.C., H.F. de Campos Velho, and N.J. Ferreira, 2005. Artificial neural network technique for rainfall forecasting applied to the Sao Paulo region. Journal of Hydrology 301(1), 146-162.

Wagener, T., and H.V. Gupta, 2005. Model identification for hydrological forecasting under uncertainty. Stochastic Environmental Research and Risk Assessment, 19(6), 378-387. 
600 Wei, S., Song, J., and Khan, N. I, 2012. Simulating and predicting river discharge time series 601 using a wavelet-neural network hybrid modelling approach. Hydrological Processes, $602 \quad$ 26(2), 281-296.

603 Yeh, W.W-G. and Yoon, Y.S., 1976. A systematic optimization procedure for the identification 604 of inhomogeneous aquifer parameters. In Advances in Groundwater Hydrology; ed. Z.A 605 Saleen, 72-82. Middleburg, Virginia: American Resource Association.

606 Yeh, W.W-G., and Yoon, Y.S, 1981. "Aquifer parameter identification with optimum dimension 607 in parameterization." Water Resources Research, 17(3), 664-672.

608 Yates, R.D., and D.J. Goodman, 1999. Probability and stochastic processes. John Wiley \& Sons 609 USA.

610 Zhang, X., Liang, F., Srinivasan, R., Van Liew, M., 2009. Estimating uncertainty of streamflow 611 simulation using Bayesian neural networks. Water Resources Research 45(2), W02403. 


\section{Table Captions:}

614 Table 1: ANN ensemble methods for prediction

615 Table 2: Maximum error and the coefficient of determination $\left(\mathrm{R}^{2}\right)$ for "in-sample" and "out616 sample" data sets, using the Best ANN (Tansig-6) and four ANN combination methods. 


\section{Figure Captions:}

618 Figure 1: A schematic of a typical multilayer perceptron ANN structure.

619 Figure 2: Map of the Maku area. The $\mathrm{x}$ and y coordinates are the UTM (meter) zone 83.

620 Figure 3: BIC, $Q_{p, q}$ and model weights using Occam's window and variance window using 18

621 ANN models.

622 Figure 4: Comparison between different variance window sizes in predicting "out-sample"

623 fluoride concentrations: (a) Occam's window ( $\alpha=1.0)$, (b) $1 \sigma$ variance window with $(\alpha=0.404)$,

624 (c) $2 \sigma$ variance window $(\alpha=0.202)$ and (d) $4 \sigma$ variance window with $(\alpha=0.101)$.

625 Figure 5: BMA tree of posterior model probabilities and conditional posterior model 626 probabilities.

627 Figure 6: Fluoride estimation variances for the "out-sample" data set. The variances from bottom

628 to top are from uncertainty in ANN weights and biases, from uncertainty in the number of hidden

629 layer nodes and from uncertainty in the hidden layer activation function.

630 Figure 7: One standard deviation error of the "out-sample" data set for (a) the best ANN (Tansig-

631 6) and (b) the hierarch model.

632 Figure 8: (a) The estimated mean fluoride concentration map and (b) the standard deviation map 633 of the estimated fluoride concentration by the hierarch model. 


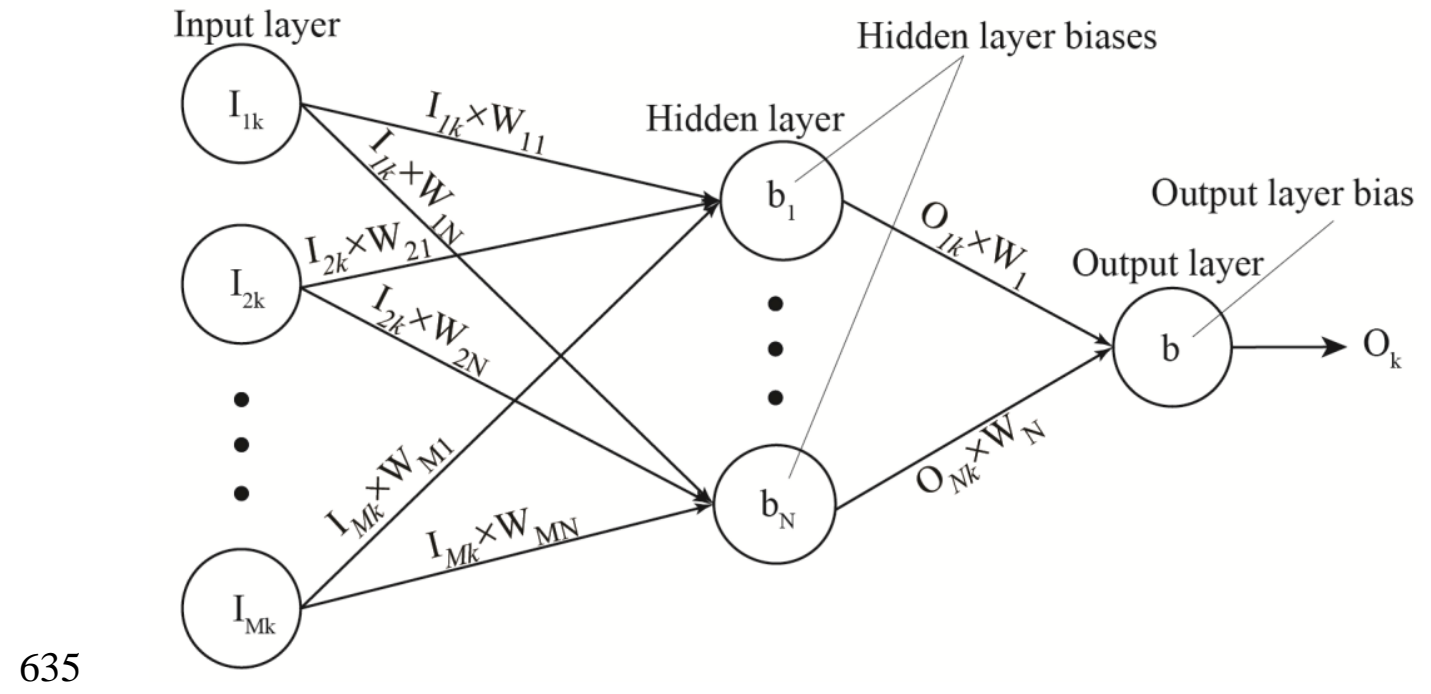

636 Figure 1 
637

638

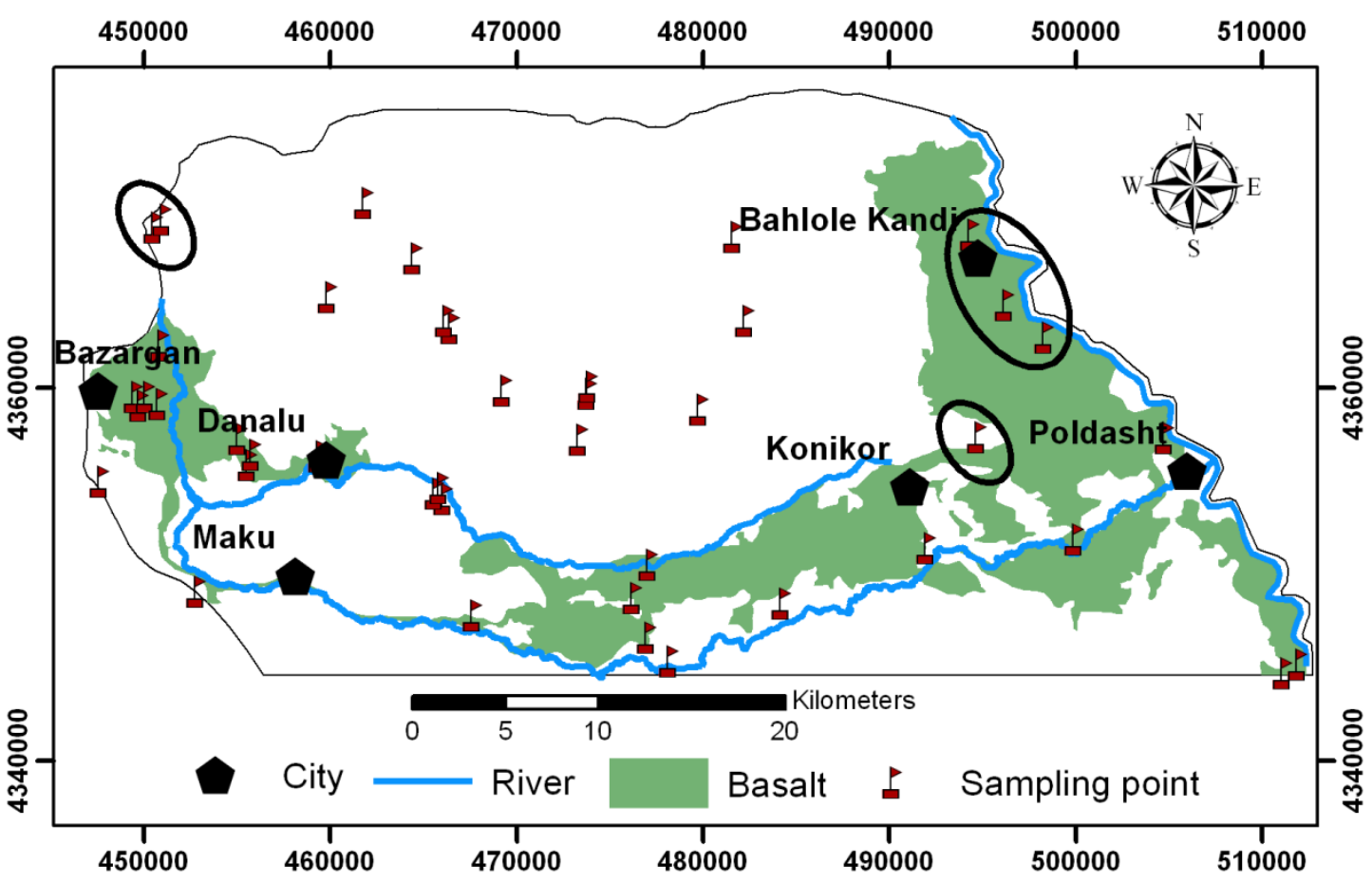

639 Figure 2 


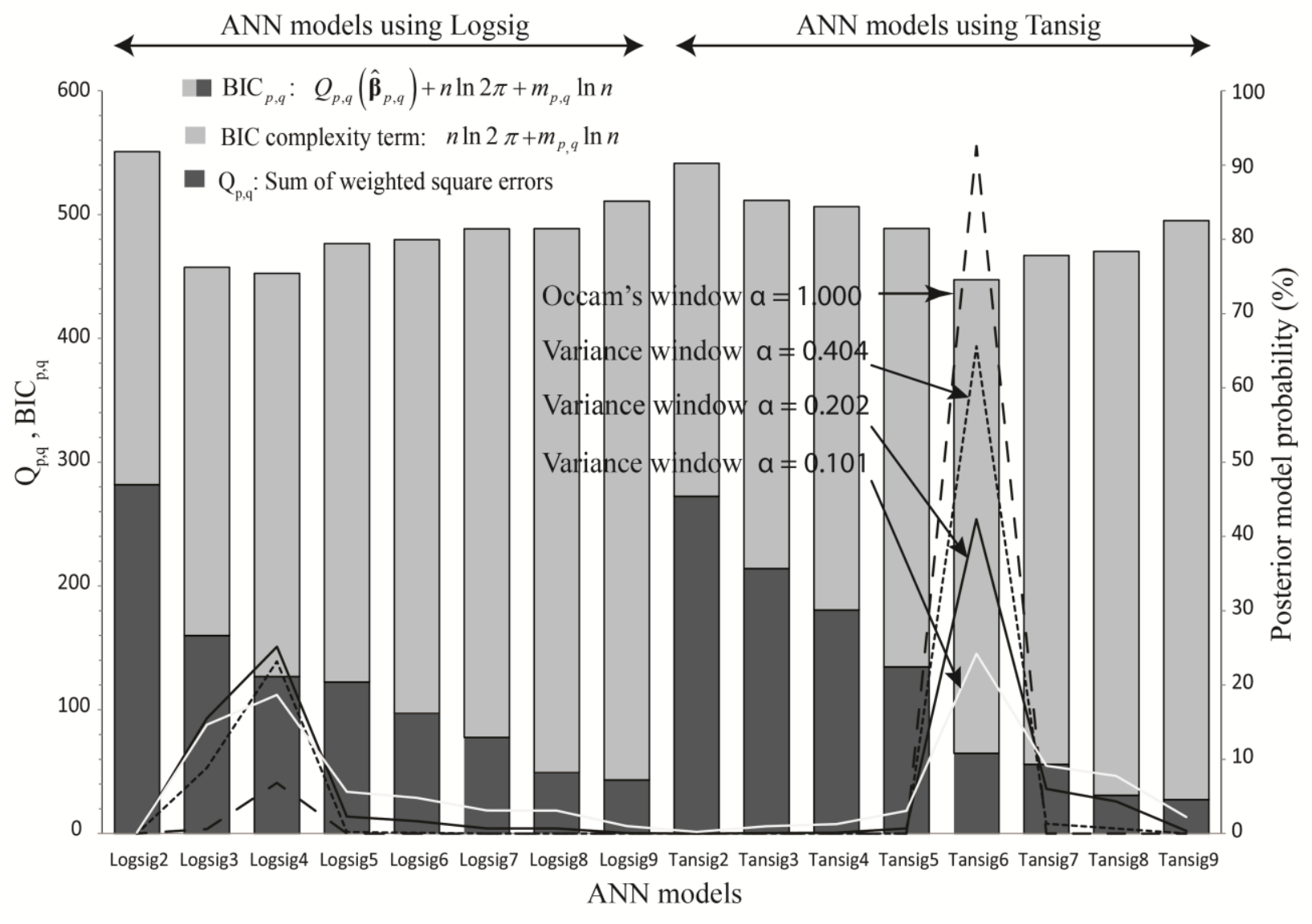

641 Figure 3 

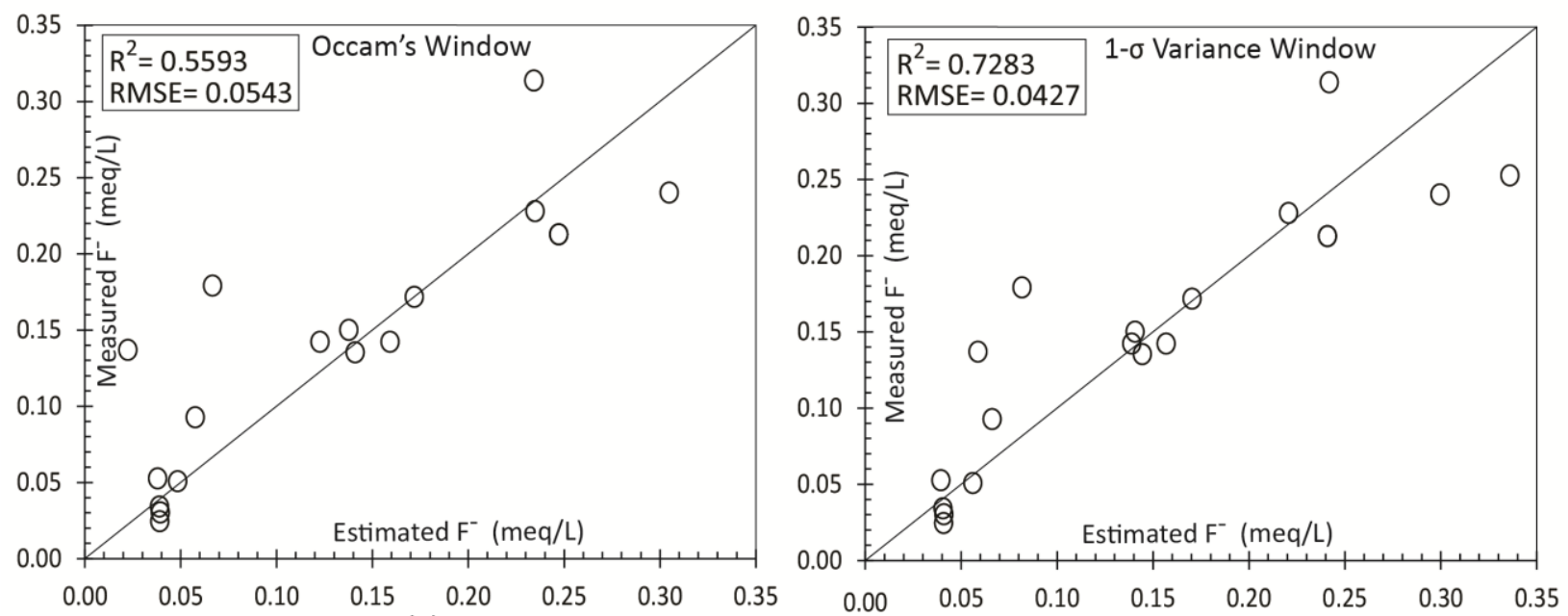

(a)

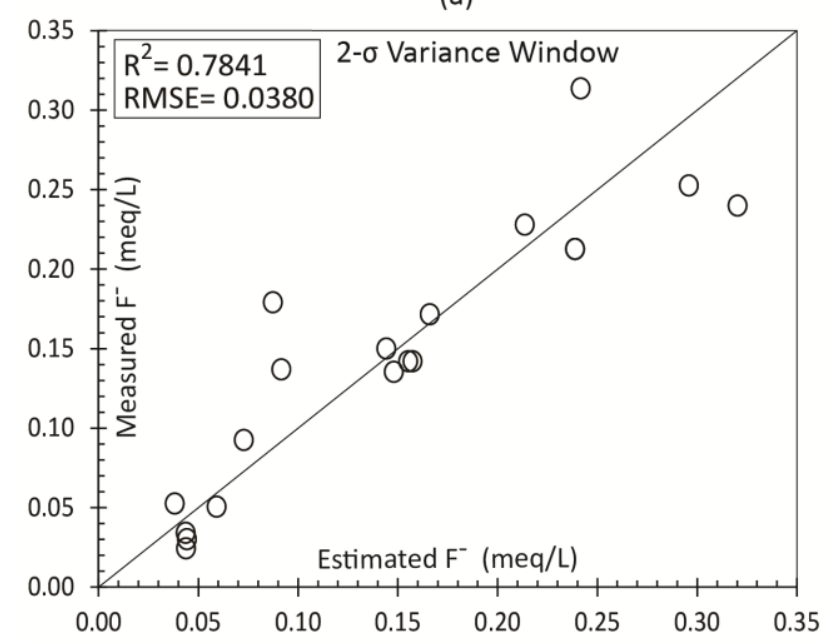

642

(c)

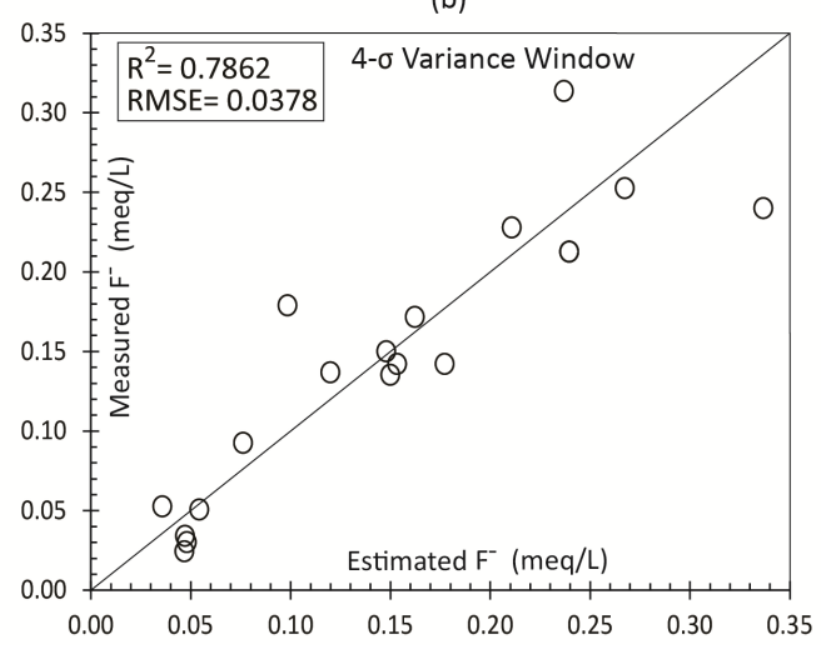

(d)

\section{Figure 4}



Hierarch
100 ------ Conditional posterior model probability
(100) - - - - - Posterior model probability

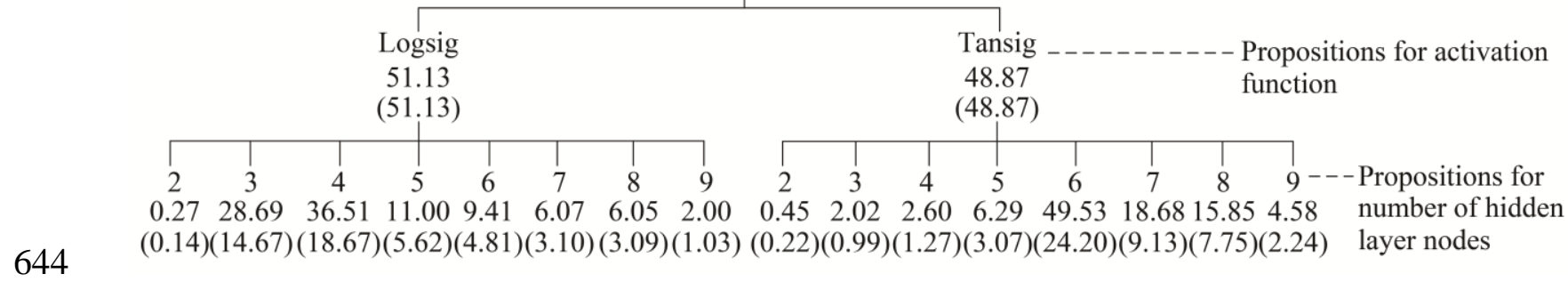

\section{Figure 5}




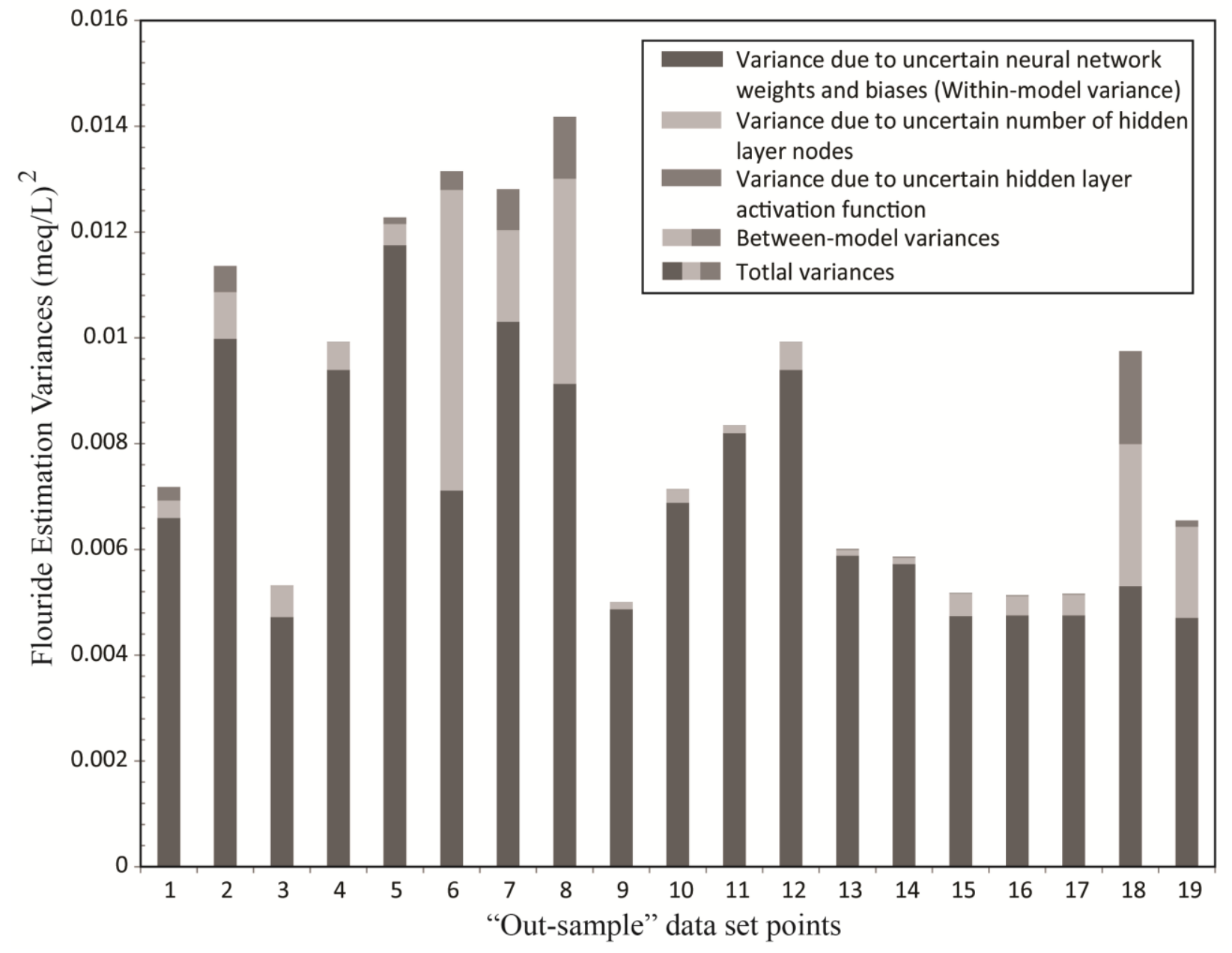

647 Figure 6 


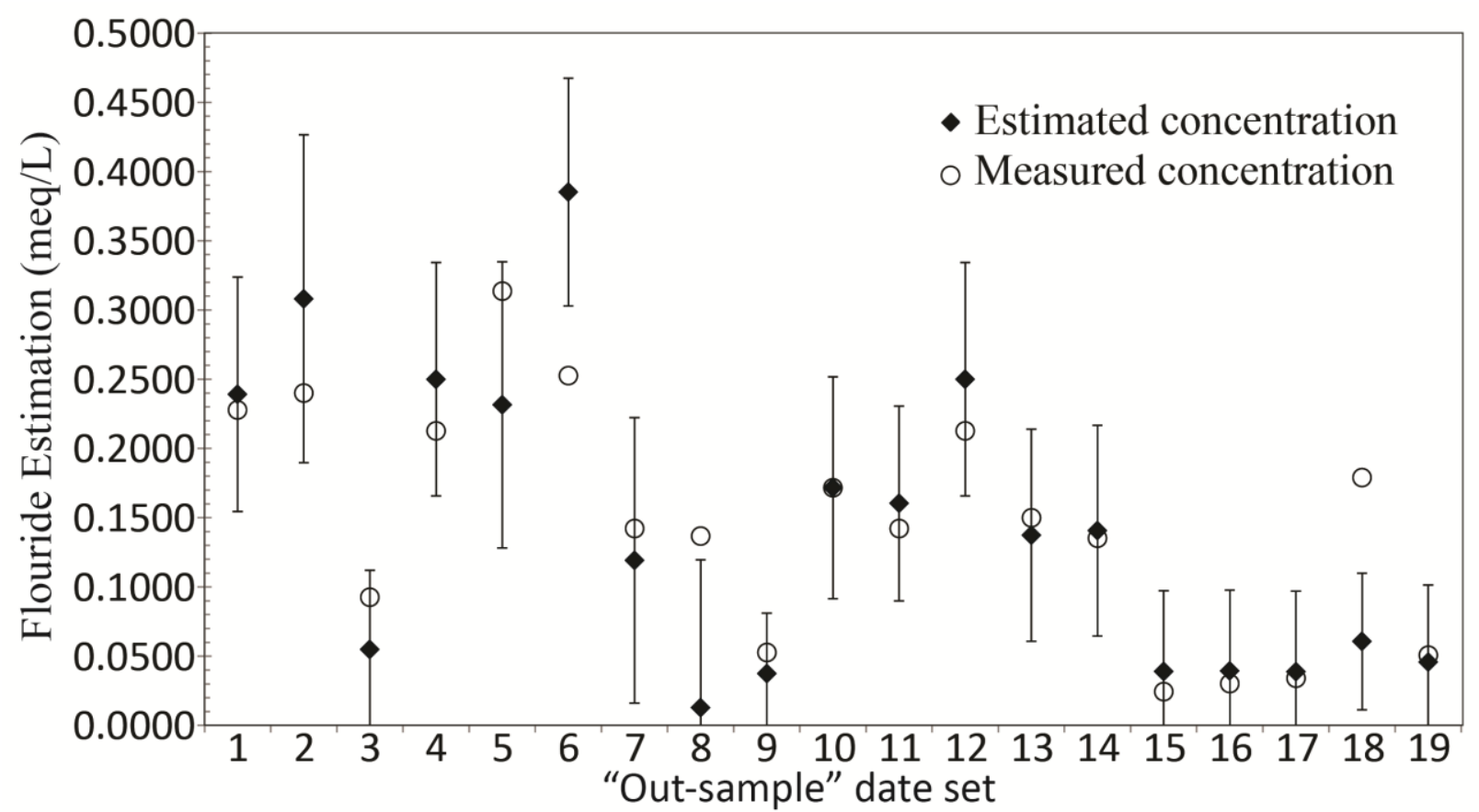

(a)

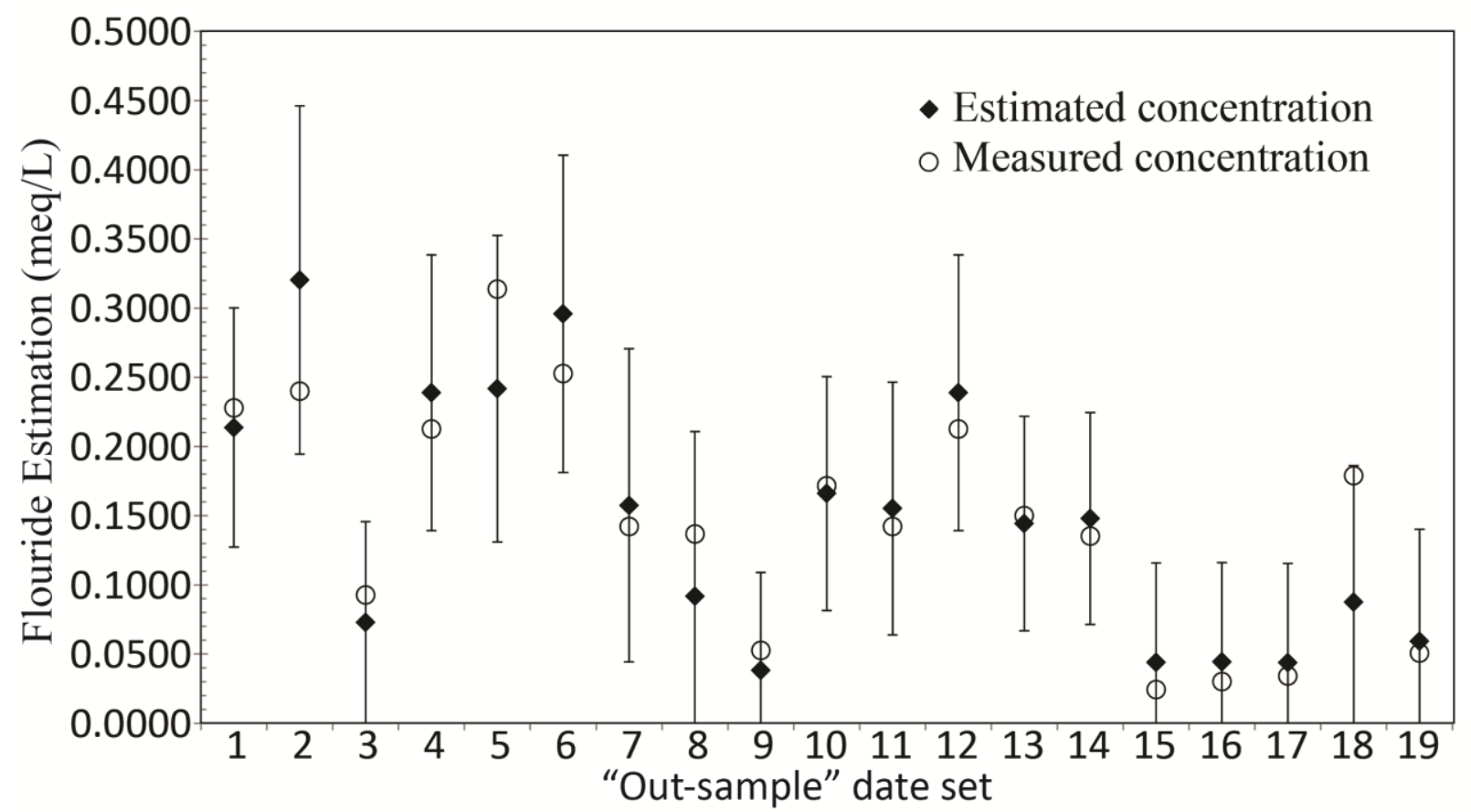

648

(b)

$649 \quad$ Figure 7 


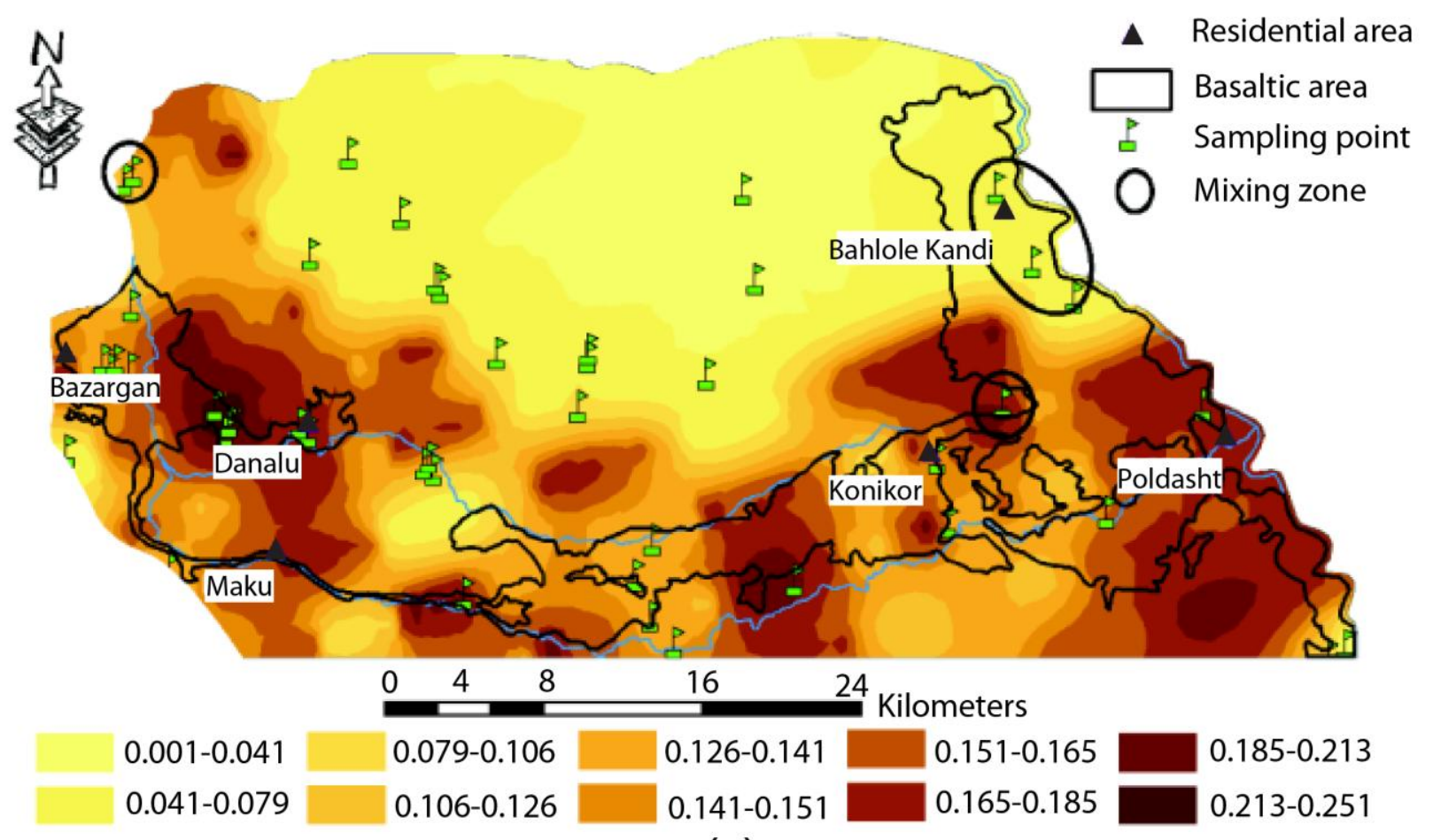

(a)

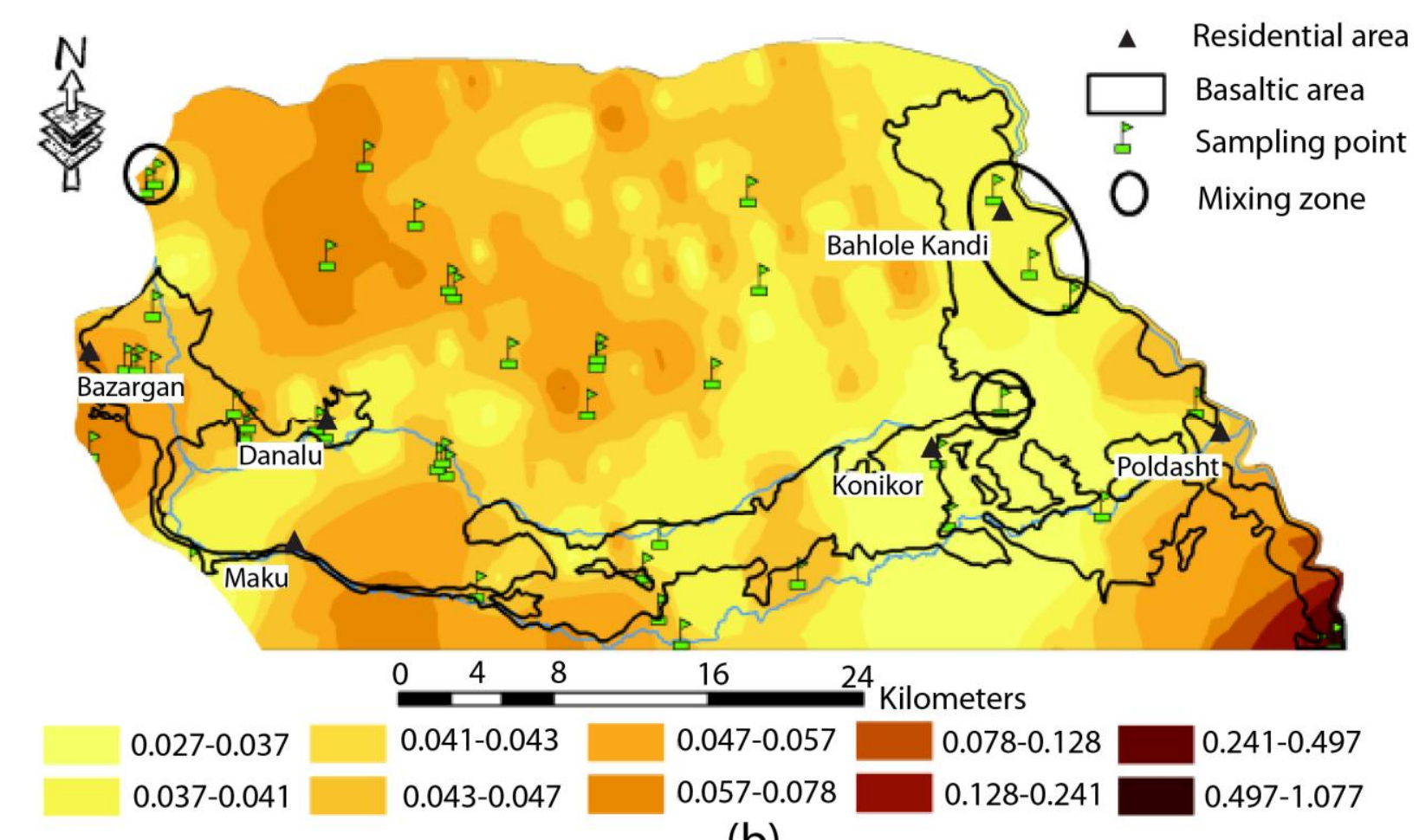

(b)

651 Figure 8 
652 Table 1: ANN ensemble methods for prediction

\begin{tabular}{|c|c|c|c|}
\hline Ensemble method & $\begin{array}{c}\text { Ensemble prediction } \\
\text { equation }\end{array}$ & Ensemble weights & References \\
\hline HBMA-NN & Equation (17) & Equation (16) & This study \\
\hline $\begin{array}{l}\text { CM (simple } \\
\text { averaging) }\end{array}$ & $F=\sum_{i=1}^{N} W_{i} \times F_{i}$ & $W_{1}=\cdots=W_{N}=\frac{1}{N}$ & $\begin{array}{l}\text { (Perrone and Cooper, } \\
\text { 1993; Wong et al., 2000) }\end{array}$ \\
\hline $\begin{array}{l}\mathrm{CM} \text { (weighted } \\
\text { averaging) }\end{array}$ & $F=\sum_{i=1}^{N} W_{i} \times F_{i}$ & $\begin{array}{l}W_{1}, W_{2}, \cdots, W_{N} \text { are } \\
\text { optimized to provide } \\
\text { the best fit to the "in- } \\
\text { sample" data set. }\end{array}$ & $\begin{array}{l}\text { (Chen and Lin, 2006; } \\
\text { Kadkhodaie-Ilkhchi et } \\
\text { al., 2009; Labani et al., } \\
\text { 2010) }\end{array}$ \\
\hline SCM & $F=b+\sum_{j} W_{j} \times f_{1}\left(b_{j}+\sum_{i=1}^{N} W_{i j} F_{i}\right.$ & $\begin{array}{l}b, b_{j}, W_{j} \text { and } W_{i j} \text { are } \\
\text { optimized by a } \\
\text { supervised ANN to } \\
\text { provide the best fit to } \\
\text { the "in-sample" data } \\
\text { set. }\end{array}$ & $\begin{array}{l}\text { (Drucker, 1997; Hu and } \\
\text { Tsoukalas, 2003; Nadiri } \\
\text { et al., 2013) }\end{array}$ \\
\hline
\end{tabular}


655 Table 2: Maximum error and the coefficient of determination $\left(\mathrm{R}^{2}\right)$ for "in-sample" and "out656 sample" data sets, using the Best ANN (Tansig-6) and four ANN combination methods.

\begin{tabular}{|c|c|c|c|c|c|c|}
\hline \multirow{2}{*}{ Model } & \multicolumn{3}{|c|}{ "In-sample" } & \multicolumn{3}{|c|}{ "Out-sample" } \\
\hline & Max Error & RMSE & $\mathrm{R}^{2}$ & Max Error & RMSE & $\mathrm{R}^{2}$ \\
\hline Tansig-6 & 0.0596 & 0.0413 & 0.9608 & 0.1326 & 0.0582 & 0.4946 \\
\hline Hierarch & 0.0370 & 0.0349 & 0.9720 & 0.0968 & 0.0378 & 0.7862 \\
\hline CM (simple averaging) & 0.0472 & 0.0390 & 0.9650 & 0.0983 & 0.0391 & 0.7656 \\
\hline CM (weighted averaging) & 0.0247 & 0.0200 & 0.9908 & 0.1179 & 0.0525 & 0.5885 \\
\hline $\mathrm{SCM}$ & 0.0237 & 0.018 & 0.9926 & 0.1007 & 0.0511 & 0.6094 \\
\hline
\end{tabular}

657

658 